\title{
BMJ Open Study protocol for the road to hierarchical diabetes management at primary care (ROADMAP) study in China: a cluster randomised controlled trial
}

\author{
Weiping Jia, ${ }^{1,2}$ Puhong Zhang, ${ }^{3,4}$ Nadila Duolikun, ${ }^{3}$ Dalong $Z$ hu, ${ }^{5} \mathrm{Hong} \mathrm{Li},{ }^{6}$ \\ Yuqian Bao, ${ }^{1}$ Xian $\mathrm{Li},{ }^{4,7}$ Yu Liu, ${ }^{8}$ ROADMAP study group
}

To cite: Jia W, Zhang $P$, Duolikun N, et al. Study protocol for the road to hierarchical diabetes management at primary care (ROADMAP) study in China: a cluster randomised controlled trial. BMJ Open 2020;10:e032734. doi:10.1136/ bmjopen-2019-032734

- Prepublication history and additional material for this paper are available online. To view these files, please visit the journal online (http://dx.doi. org/10.1136/bmjopen-2019032734).

WJ and PZ are joint first authors.

Received 02 July 2019

Revised 04 October 2019

Accepted 26 November 2019

Check for updates

(C) Author(s) (or their employer(s)) 2020. Re-use permitted under CC BY-NC. No commercial re-use. See rights and permissions. Published by BMJ.

For numbered affiliations see end of article.

Correspondence to Professor Weiping Jia; wpjia@sjtu.edu.cn

\section{ABSTRACT}

Introduction Diabetes management in primary care remains suboptimal in China, despite its inclusion in the essential public health service (EPHS). We aimed to evaluate the effectiveness of a mobile health (mHealth) based and three-tiered diabetes management system in diverse Chinese contexts.

Methods and analysis This is a cluster randomised controlled trial, named road to hierarchical diabetes management at primary care (ROADMAP). 19008 patients with type 2 diabetes (T2D) were recruited from primary care clinics in 864 communities across 144 counties/districts of 24 provinces. Eligible participants were adult patients diagnosed with $\mathrm{T} 2 \mathrm{D}$ and registered for diabetes management in communities. Patients within the same communities (clusters) were randomly allocated into the intervention or control arm for 1 year in a 2:1 ratio. The control arm patients received usual care as EPHS packaged: at least four blood glucose (BG) and blood pressure (BP) tests, and lifestyle and medication instruction, yearly, from primary care providers. The intervention arm patients received at least two BG and one BP tests, monthly, and lifestyle and treatment instruction from a three-tiered contracted team. A mHealth platform, Graded ROADMAP, enabled test results uploading and sharing, and patient referral within the team. The intervention participants will be further divided into basic or intensive intervention group according to whether they were actively using the Your DoctorApp. The primary outcome is the BG control rate with glycated haemoglobin $(\mathrm{HbA} 1 \mathrm{c})<7.0 \%$. Secondary outcomes include control rates and changes of ABC (HbA1c, BP and low-density lipoprotein cholesterol) and fasting $B G$, hypoglycaemia episodes and health-related quality of life (EuroQol (EQ-5D)).

Ethics and dissemination The trial has been approved by the Institutional Review Board at Shanghai Sixth People's Hospital. Findings on the intervention effectiveness will be disseminated through peer-reviewed journals, conference presentations and other relevant mechanisms.

Trial registration number ChiCTR-IOC-17011325.

\section{INTRODUCTION}

Type 2 diabetes (T2D) imposes major morbidity, mortality and financial burden

\section{Strengths and limitations of this study}

- This is a large-scale cluster clinical trial being implemented in diverse primary care settings of most provinces in China. The sample size allows for a formative evaluation of a complex service intervention overall, and in subgroups of developed and lessdeveloped, urban and rural regions.

- A standardised 4-in-1 comprehensive management package is designed to empower the capabilities of primary care to improve the overall diabetes management in real-world settings beyond this study.

- The intervention delivery is supported by two designated mobile-based information system (Graded ROADMAP, Your Doctor), recording the condition of participants, receipt of services and health education and communications, to ensure the continuum of care with secure access.

- A centrally distributed, portable instrument was applied for measuring the primary outcome, glycated haemoglobin $(\mathrm{HbA} 1 \mathrm{c})$, playing a role as a centralised laboratory, thus ensuring the comparability of $\mathrm{HbA} 1 \mathrm{C}$ level.

- We suggest caution in generalising any intervention benefits to the broader diabetes population, since the recruitment is limited to people with diagnosed type 2 diabetes registered for receiving services under the essential public health service package in China.

across all health systems. ${ }^{1-3}$ Globally, there are an estimated 425 million people with T2D and one fourth of them reside in China where enormous challenges of diabetes management exist given the large population across diverse settings. ${ }^{1-6}$ Although an essential public health services (EPHS) package, with annual increases in governmental subsides ${ }^{7}$ was introduced to provide universal access to services for patients with chronic diseases in 


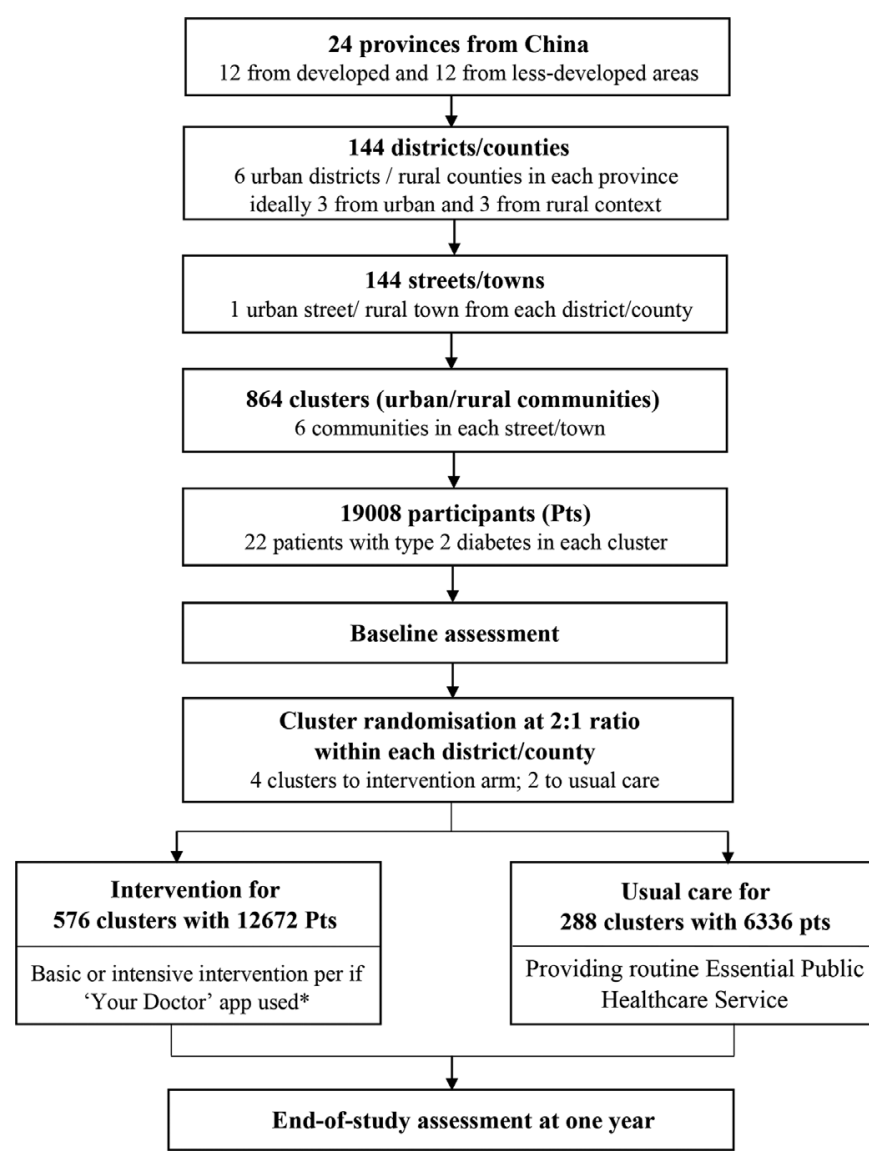

Figure 1 Flow chart of the ROADMAP study. (1) Provinces in the upper half of provincial gross domestic product per capita in fiscal year of 2017 are classified into developed areas, and those at lower half are less-developed areas; urban or rural areas are classified as per national administrative area category in 2017. (2) Your Doctor app is available for those participants who possess a smartphone and capable of using applications, enabling health education and real-time communication with doctors. Patients who have used 'Your Doctor' app will be regarded as receiving intensive intervention.

primary care in China in $2009,{ }^{8}$ considerable gaps in care remain.

The EPHS packaged services are delivered by primary care providers through community healthcare service (CHS) centres (sub-district level) and stations (community level) in urban areas, and township hospitals and village clinics in rural areas. Management of those with T2D is an explicit focus of the EPHS package. ${ }^{49}$ By 2017 , there were over 9100 CHS centres, 25500 CHS stations, 36500 township hospitals and 632000 village clinics in operation nationwide. ${ }^{10}$ (Further structural details of the primary care service delivery in China is available in online supplementary appendix 1.) Patients registered for T2D management of EPHS are entitled to at least four free blood glucose (BG) tests, and consultations for treatment and lifestyle advice each year. ${ }^{7}$ Despite an increasing number of patients receiving T2D management services, from 18.5 million in 2011 to 31.2 million in $2017,{ }^{11}$ fewer than $40 \%$ of patients had adequate BG control (glycated haemoglobin $(\mathrm{HbAlc})<7.0 \%),{ }^{12}$ with only $6 \%$ achieving optimal control of combined ABC (HbAlc, blood pressure (BP) and low-density lipoprotein cholesterol (LDLC) ) targets. ${ }^{13}$

Achieving effective T2D management is heavily dependent on an organised and responsive primary care system. ${ }^{1415}$ Inadequate capacity of primary healthcare providers, inefficient resource utilisation and, most importantly, fragmented delivery of care have been identified as major challenges in primary care that have potentially impeded the improvement in diabetes control. ${ }^{416-21}$ It is reported that over $25 \%$ of doctors at CHS centres and $45 \%$ at township hospitals have not reached the educational requirement for a licensed assistant doctor. Twelve percent of village clinic doctors were below the required education level for their position and one-third of primary healthcare providers do not receive annual training, a requirement of the authorities. ${ }^{4}$ A skills shortage as a result of inadequate education and training contributes to poorer quality care, partially explaining why patients tend to bypass primary care facilities and present to hospitals. ${ }^{22}$ Also, the fragmentation of the service delivery system and a lack of interoperable information systems have hindered the integration of the primary care and specialty care in secondary and tertiary hospitals, obstructing the coordination and continuum in care. ${ }^{4}$

In 2015, national guidelines promoting a graded diagnosis and treatment system were introduced ${ }^{23}$ in China, in line with the Healthy China 2030 policy $^{24}$ to establish a cooperative, hierarchical health service system. While evidence on the effectiveness of mobile health (mHealth)-based strategies that involve service delivery design to improve diabetes management is demonstrated, ${ }^{25-31}$ its adaptability in primary care facilities varies across different economic contexts and regions. ${ }^{32}{ }^{33} \mathrm{We}$ therefore outline the protocol for a study with the aim of evaluating the effectiveness of a mHealth-based, servicedelivery intervention in a cluster randomised controlled trial called road to hierarchical diabetes management at primary care (ROADMAP). The intervention follows the recommendations from the Chinese Diabetes Society (CDS) guideline ${ }^{34}$ to empower primary care providers in a collaborative tiered care system with mHealth-based health information and referral management system to deliver standardised services for patients with T2D in China.

\section{METHODS AND ANALYSIS}

\section{Trial design and study contexts}

ROADMAP is a community-based, cluster randomised controlled trial, comparing the effectiveness between the proposed intervention and the usual care on diabetes management in diverse primary care settings (figure 1). Within the intervention arm, participants will be further divided into basic and intensive intervention subgroups, based on whether an optional health 
Table 1 Eligibility criteria for study sites in ROADMAP study

\begin{tabular}{|c|c|}
\hline $\begin{array}{l}\text { Study site at } \\
\text { different levels }\end{array}$ & Eligibility criteria description \\
\hline Province & $\begin{array}{l}\text { At least one provincial principal investigator with high impact in diabetes research is available and } \\
\text { approved by ROADMAP working group. } \\
\text { Provinces planning to develop or have had established a primary healthcare service based on } \\
\text { electronic referral system will be excluded due to a potential technological conflict. }\end{array}$ \\
\hline District/county & $\begin{array}{l}\text { County hospitals should be the main healthcare providing institution rather than tertiary hospitals to } \\
\text { avoid affecting the process of patient referral and future impact evaluation. } \\
\text { There is no generalised mHealth-based referral system in place, and an adequate level of community } \\
\text { healthcare facilities and service is available. } \\
\text { Local health authorities are aware of, support and are willing to be involved with the trial, anticipating } \\
\text { to become an exemplary centre of diabetes management. } \\
\text { There is at least one diabetes specialist able to take the role of county principal investigator/doctor to } \\
\text { deliver training, support treatment and receive referral, as well as one county coordinator to facilitate } \\
\text { trail implementation and fidelity assurance, also manage local study funds. } \\
\text { There are at least one eligible sub-districts/towns available within each district/county. } \\
\text { Legitimate healthcare facility that can provide formal invoices for all trial-incurred transactions. }\end{array}$ \\
\hline Sub-district/town & $\begin{array}{l}\text { The methods and instruments are readily available to perform high-quality laboratory tests, including } \\
\text { plasma glucose test, routine urinalysis, lipid profile, blood creatinine, liver function and ECG; having a } \\
\text { non-mydriatic fundus camera is preferred. } \\
\text { Insulin is available to store essential medicines. } \\
\text { All participating doctors possess smartphones and are capable of using applications. } \\
\text { There are six potential eligible communities available within each sub-district/town. } \\
\text { T2D patients registered in the essential public healthcare service system have documented general } \\
\text { information and contact details. }\end{array}$ \\
\hline Community & $\begin{array}{l}\text { More than } 35 \text { registered patients with diagnosed T2D in community. } \\
\text { The community is not involved in any other clinical trial. There is no mHealth-based referral system in } \\
\text { use. } \\
\text { All participating doctors possess and are capable of using smartphone (iOS } 7 \text { or Android } 4.4 \text { and } \\
\text { above). No difficulty in installing and using applications. } \\
\text { Community doctors are willing to participate in the trial. }\end{array}$ \\
\hline
\end{tabular}

mHealth, mobile health; ROADMAP, road to hierarchical diabetes management at primary care; T2D, type 2 diabetes.

education smartphone application is used. The trial will be conducted in over 800 communities in more than 20 provinces of China. To better integrate clinical services and primary care, the implementation of the trial involves three tiers of healthcare institutions: regional medical centres (tier III: hospitals at urban district/rural county level), primary care centres (tier II: CHS centres at urban sub-district/township hospitals at rural town level) and their subordinate primary care clinics (tier 1: urban CHS stations and rural village clinics at level of community (also refer to village in rural area hereinafter)).

\section{Study sites selection and participants}

Table 1 details the inclusion/exclusion criteria for study sites selection at multiple levels, including provincial, district and county level, sub-district and town level, and community level. 24 provinces will be selected nationally. In general, 6 counties, and 6 of its subordinate communities from each county will be selected in each province. In each community (cluster), an average of 22 participants will be selected at random via a purpose-built website from a full list of T2D patients, normally with over 50 registrants, from local EPHS recipient registry in each community.

Participants will be patients with diagnosed T2D and registered for receiving EPHS in the participating communities. Individuals are eligible for enrolment if they meet following inclusion criteria: aged 18-75 years; residing in the community for the last 6 months with no plan of relocating; voluntary participation with informed consent. Patients will be excluded if: they had severe physical or psychological problems; were unable to attend the site visit; unable to consciously answer questions; were women in the process of, or planning for, pregnancy or breast feeding; and those who have participated in any other clinical trials within the last 6 months.

\section{Randomisation}

Randomisation will be performed centrally via a passwordprotected, encrypted smartphone application interface, stratified by provincial ranking of gross domestic product (GDP) per capita in fiscal year of $2017,{ }^{35}$ with upper and lower half categorised as developed and less-developed, respectively, and locality including urban and rural areas as per national administrative area category in 2017. 
Following baseline data collection, these six communities (ie, clusters) will be randomised to either receive the intervention or continue with usual care in a 2:1 ratio (ie, 4 clusters to intervention, 2 to control).

\section{Interventions}

The basic intervention consists of four elements: (1) capacity building for service providers at primary care facilities; (2) team-based contracted standardised services; (3) mHealth-based information support; and (4) reimbursement for service delivery. The basic intervention elements are elaborated in the next paragraph. There is an additional intensive component available to participants with smartphones willing to use Your Doctor. Your Doctor is a smartphone application supporting health education (examples are available in the online supplementary appendix 2) and treatment instruction through real-time within-app interaction between the patients and their contacted doctors. Active users are defined as those with a login to Your Doctor at least four times throughout the 1-year study period. At the end of study, participants in the intervention arm will be divided into two groups (basic or intensive), based on their level of activity on Your Doctor.

\section{Capacity building}

Two compulsory structured training sessions will be held at the provincial and county level. The initial provinciallevel training session is for doctors from participating county hospitals. This will be followed by training at the district/county level, where the qualified trainers provide training for the sub-district/township and community doctors. The training materials were developed by the working group based on current diabetes guidelines, with the purpose of upskilling service providers by addressing theoretical and operational barriers to T2D management and treatment in primary care settings. The contents include: (1) diabetes epidemiology and economic burden; (2) classification and diagnosis of diabetes; (3) hierarchical diagnosis and treatment of diabetes; (4) diabetes lifestyle intervention; (5) diabetes medication; (6) BG monitoring; (7) diabetic retinopathy; (8) diabetic nephropathy; (9) diabetic peripheral neuropathy; and (10) diabetic lower limb vascular diseases. The trainings are standardised to ensure the service would be delivered in the same manner. A package of training materials, including a set of Powerpoint slides and videos, will be sent to all doctors in the intervention arm for periodic review. Investigators in the control arm will only attend trainings for subject recruitment and data collection procedures for baseline and end-of-study assessments.

\section{Team-based contracted services}

A three-tiered care team will be assembled for each participating community. The team members comprise of three doctors, respectively, from the primary care clinic, primary care centre and regional medical centre. The primary care doctors will play a key role in routine contacting, monitoring and evaluation, service delivery and lifestyle instructions to the patients. Doctors in the regional medical centres will provide further consultation or treatment if referred to. A service contract will be signed between the care team doctors and participants to acknowledge the engagement and inform stakeholders' responsibilities. (A full list of contracted services are available in the online supplementary appendix 3.) The planned services are listed in table 2 with comparison to those in EPHS as usual care.

\section{mHealth-based information support}

Most intervention activities will be carried out by or through Graded ROADMAP App (some user interfaces are displayed in the online supplementary appendix 4), including medical information recording, BG/BP monitoring, processing of referral requests and responses (referral indications are available in the online supplementary appendix 5) and performance reviews. The user ends vary with the services provided by different users. Patient data are only accessible within the contracted care team to ensure the continuum in care. For intensive group patients, Your Doctor App will be used to share their $\mathrm{BG} / \mathrm{BP}$ results and interact with their doctors for better diabetes control.

\section{Reimbursement for service delivery}

Non-mandatory seasonal payments, RMB 55 per participant averagely, are suggested every 3 months to compensate care providers' extra work hours for additional service delivery and also serve as a 'fee for performance' incentive for protocol compliance. The performance of the service is appraised by the aggregated data from an algorithm incorporating BG/BP measurements and control, the number of screening for any complication of T2D and necessary referrals. The amount of the reimbursement is capped at RMB 220 Yuan for each patient per year. It is estimated to be equivalent to one-tenth of per capita public input for EPHS, which is acceptable by the government if the intervention is effective.

To sum up, with support of Graded ROADMAP, the key and fundamental intervention in our study is to offer the patients with access to more frequent BG and BP monitoring services, and followed by corresponding instructions on lifestyle modification and medication treatment.

\section{Usual care}

Participants in the control arm will continue receiving usual care provided by primary care facilities, as per EPHS regulations. For community patients with T2D, the key services provided by primary care facilities were presented in table 2. Several other related services in EPHS include developing personal health records, updating health records after follow-ups, population-based health education, health management for the elderly with annual services including lifestyle and health assessment, physical examination, and health guidance for the elderly over 65 years of age, and hypertension management. ${ }^{7}$ 
Table 2 Diabetes management related training, technical support and health services provided for intervention and control arms

\begin{tabular}{|c|c|c|}
\hline Items & $\begin{array}{l}\text { Control arm } \\
\text { (usual care per EPHS) }\end{array}$ & $\begin{array}{l}\text { Intervention arm } \\
\text { (Strengthened care in addition to EPHS) }\end{array}$ \\
\hline $\begin{array}{l}\text { Capacity building for } \\
\text { primary care providers }\end{array}$ & $\begin{array}{l}\text { Provided by local health } \\
\text { bureau, aims to implement } \\
\text { EPHS, no national standard } \\
\text { training material. }\end{array}$ & $\begin{array}{l}\text { Trained by qualified trainers using national standard training } \\
\text { materials, addressing major gaps in knowledge and skills in diabetes } \\
\text { management, including diabetic peripheral neuropathy screening. }\end{array}$ \\
\hline Technical support & $\begin{array}{l}\text { Equipped with BG meter, BP } \\
\text { monitor and body weight/ } \\
\text { height scale. Mostly equipped } \\
\text { with electronic medical } \\
\text { record system but unable to } \\
\text { communicate with local medical } \\
\text { centres. }\end{array}$ & $\begin{array}{l}\text { Equipped with (1) a portable electronic BG meter that enables the } \\
\text { test result uploaded to an electronic information platform in real } \\
\text { time; (2) Graded ROADMAP, the platform, developed by the working } \\
\text { group, acts as an electronic medical record system with features } \\
\text { of communication with electronic BG monitors and patient referral } \\
\text { among doctors in the same care team; and (3) a set of } 128 \mathrm{~Hz} \text { tuning } \\
\text { forks and } 10 \mathrm{~g} \text { nylon monofilaments used to screen peripheral } \\
\text { neuropathy. }\end{array}$ \\
\hline Basic BP monitoring & At least four times per year & Monthly or more frequently, emphasised as compulsory. \\
\hline $\begin{array}{l}\text { Diabetic peripheral } \\
\text { neuropathy screening }\end{array}$ & Not required & $\begin{array}{l}\text { At least once per year with the hypotheses that (early) knowledge } \\
\text { of diabetes complications can improve the adherence to diabetes } \\
\text { control. This was suggested to start at the beginning of intervention. }\end{array}$ \\
\hline $\begin{array}{l}\text { Diet, physical exercise } \\
\text { and medication } \\
\text { instruction }\end{array}$ & $\begin{array}{l}\text { Each time during face to face } \\
\text { visit }\end{array}$ & $\begin{array}{l}\text { Face to face, together with remote (online) communication through } \\
\text { 'Your Doctor', an App supporting health education and treatment } \\
\text { instruction through interactive real-time communication between the } \\
\text { contacted doctors and patients (intensive intervention subgroup). }\end{array}$ \\
\hline Patient referral & $\begin{array}{l}\text { Major indications: (1) BG } \\
\text { remains uncontrolled for two } \\
\text { consecutive visits; (2) adverse } \\
\text { drug reactions do not improve } \\
\text { after two consecutive visits; } \\
\text { or (3) new or aggravated } \\
\text { complications. } \\
\text { Pathway: no designated } \\
\text { pathway; patients can seek } \\
\text { service from any doctor in any } \\
\text { hospital according to their } \\
\text { wishes. }\end{array}$ & $\begin{array}{l}\text { Indications: similar to those in control arm. } \\
\text { Pathway: The referral is designed to be processed (initialed/accpeted } \\
\text { or rejected) through the Graded ROADMAP app, hypothesizing } \\
\text { that the mHealth-based referral within the contracted team would } \\
\text { improve the referral and diabetes control. This app has different } \\
\text { end-users. A primary care doctor can make the appointment for the } \\
\text { patient by selecting a referral doctor (usually the doctor in the same } \\
\text { team) and date based on the availability of upstream doctors and } \\
\text { wishes of patients. The medical record, including the trend of BG and } \\
\text { medications, could be accessed by all the authorised doctors. }\end{array}$ \\
\hline
\end{tabular}

BG, blood glucose; BP, blood pressure; EPHS, essential public health service.

Theoretically, all services provided to usual care were also delivered to the intervention arm.

\section{Outcomes}

The primary outcome is the change of BG control rate with $\mathrm{HbA1c}<7.0 \%$ between baseline and 1-year follow-up. Secondary outcomes include levels and control rates of $\mathrm{ABC}(\mathrm{HbAlc}<7.0 \%, \mathrm{BP}<140 / 80 \mathrm{~mm} \mathrm{Hg}$ and LDL$\mathrm{C}<2.6 \mathrm{mmol} / \mathrm{L}$ ) targets, and fasting $\mathrm{BG}<7.0 \mathrm{mmol} / \mathrm{L}$, episodes of hypoglycaemia $(\mathrm{BG}<3.9 \mathrm{mmol} / \mathrm{L})$ and mean changes in health-related quality of life on the EQ-5D.

\section{Data collection and management}

Table 3 outlines the procedures for collecting baseline and end-of-study data through a secure password protected mobile electronic data capture (mEDC) app.
The data collecting investigators who are unaware of the allocation are trained and not involved in intervention implementation. The primary outcome will be obtained from a centrally distributed HbA1c analyser (A1c EZ 2.0) for point-of-care testing. ${ }^{36}$ Anthropometric measurements are taken with the patient barefoot in light clothing. Standard laboratory tests of blood and urine samples, including fasting glucose, lipid profile, creatinine, and kidney function, will be performed by qualified laboratories at regional medical centres. Two extra blood samples (blood biochemistry analysis and HbA1c) and one urine sample from each village will be audited for accuracy. The mEDC has embedded features to allow logic checks and photography of source documents to support real-time data collection process overseen. 
Table 3 Data collection outline of ROADMAP study

\begin{tabular}{llll}
\hline Assessment description & $\begin{array}{l}\text { Patient } \\
\text { screening }\end{array}$ & $\begin{array}{l}\text { Baseline } \\
\text { assessment }\end{array}$ & $\begin{array}{l}\text { End-of-study } \\
\text { assessment }\end{array}$ \\
\hline Informed consent & $\mathrm{X}$ & & \\
\hline Eligibility & $\mathrm{X}$ & $\mathrm{X}$ & $\mathrm{X}$ \\
\hline Reasons for non-participation & $\mathrm{X}$ & $\mathrm{X}$ & \\
\hline Demographics, socio-economic status & & $\mathrm{X}$ & $\mathrm{X}$ \\
\hline Medical history, diabetes-related complications screenings & & $\mathrm{X}$ & $\mathrm{X}$ \\
\hline Anthropometric measurements, physical examination, vital signs & & $\mathrm{X}$ & $\mathrm{X}$ \\
\hline Diabetes self-management & & $\mathrm{X}$ & $\mathrm{X}$ \\
\hline Costs of healthcare use & & $\mathrm{X}$ & $\mathrm{X}$ \\
\hline Medications and treatment adherence & & $\mathrm{X}$ & $\mathrm{X}$ \\
\hline Laboratory results & & $\mathrm{X}$ & $\mathrm{X}$ \\
\hline Quality of life questionnaire & & & \\
Loss-to-follow-up/drop-out questionnaire & & \\
\hline
\end{tabular}

Data collection, storage and reporting conforms to China's privacy laws. Individual participants are encrypted and de-identified. Feedback and queries raised on data integrity, authenticity and accuracy, as well as schedule management, are achieved using unique identifier reporting. Findings raised from this study will be reported without individual participant identification.

\section{Project management}

A trial monitoring website presents aggregated regional routinely collected data on community/township/ county/provincial levels of intervention clusters to allow researchers, investigators and doctors in the intervention group to monitor the intervention fidelity and study progress. Central and on-site monitoring of routinely collected data is undertaken by a project management team. All required qualification assessments for institutions/personnel involved in the study, including their training and investigative procedures, are recorded and photographed for reference. During each site visit, local investigators and trial staff assist research monitoring in verifying patient eligibility, data authenticity and implementation consistency, schedule compliance, and in providing all relevant source documents.

\section{Sample size estimation}

This study aims to recruit 19008 patients with T2D from 864 communities in 24 provinces in mainland China, which equates to 22 patients from each community. Accounting for potential loss to follow-up of $14 \%$ in patients, a sample size of 16416 participants (10944 in intervention and 5472 in control groups, with 2:1 ratio) at 1 year will provide a power of $89 \%$ (2-sided $\alpha 0.05$ ) to detect a $\geq 5 \%$ absolute increase in the primary outcome in the intervention group. The sample size calculation assumes that $40 \%$ of participants will have well-controlled HbAlc $(<7 \%)$ at the end of the study in the control group, ${ }^{13}$ with an intra-class correlation coefficient of
0.15 based on our previous Observational Registry for Basal Insulin Treatment (ORBIT) study. ${ }^{37}$ Furthermore, assuming that $50 \%$ (5472) of participants in the intervention group will become the active users of the Your Doctor App at their discretion, thus forming an intensive intervention subpopulation (ie, a smaller cluster size of 9 participants), which will provide $86 \%$ and $99 \%$ power to detect absolute increases of $5 \%$ and $10 \%$ of HbAlc control, respectively, compared with the control group.

\section{Outcome analysis}

All analyses will be conducted at the participant level following the intention-to-treat principle. The primary endpoint, adequate control of HbA1c at 1 year, will be compared between all intervention groups and all control groups. The primary analysis of the intervention effect will be conducted by using a log-binomial regression with generalised estimating equations to account for clustering within communities with adjustment of baseline HbA1c as a continuous covariate. Secondary analyses will include (1) covariate-adjusted analyses, on the primary outcome, (2) comparison of the intensive versus basic intervention groups by exploring if there is an additional effect from intensive intervention and (3) subgroup analyses. A detailed statistical analysis plan is anticipated to be published prior to database lock or attached to the main paper.

\section{Process evaluation}

Given the widespread geographical coverage of participating sites, the staffing model and implementation of the ROADMAP intervention may differ across different regions. A process evaluation will be conducted during implementation to assess the compliance of each intervention component and identify the implementation barriers and facilitators, as well as the acceptability to improve the implementation quality and intervention fidelity. A mixed-methods approach will be adopted using 
routinely collected data (eg, BG/BP monitoring data) and stakeholder interviews. The lessons from different regions with different staffing models will be helpful to guide the future intervention diffusion to different primary care settings.

\section{Economic evaluation}

The economic evaluation will assess cost-effectiveness/ utility from a health sector perspective, with a trial-based component and beyond-trial modelled evaluation of long-term costs and benefits, assessed using an incremental cost-effectiveness ratio. The within-trial cost will include intervention costs on trainings of staff, BG testing device and consumables, information system development and maintenance and service reimbursement, as well as healthcare use during the trial period. The effectiveness/utility will be according to the change on HbA1c within 1 year, and modelled on health-related quality of life based on the literature for the relationship of HbAlc reduction and prevention of T2D complications and improved health-related quality of life. Sensitivity analyses will be carried out to examine different scale-up scenarios in different strata.

\section{Governance}

Execution of the study is managed by a working group under the supervision of a steering committee, chaired by the leading principal investigators (PI) and representatives of the CDS and central government. The working group comprises delegates of China representative office of The George Institute for Global Health, CDS, Bethune Charity Foundation and provincial investigators.

\section{Trial status}

After the launch of the pilot phase in March 2017, the first provincial site for the main study was initiated on 2 June 2017. Up to December 2018, 19149 participants had been validly recruited from 864 communities in 144 districts/counties in 25 participating provinces. One more province was recruited due to the difficulty in finding sufficient eligible district/county hospitals as planned in 24 provinces. Baseline assessments are complete and the intervention has commenced in 576 communities from 25 provinces. The final end-of-study assessment is due in October 2019 and database lock is anticipated for December 2019.

\section{Patient and public involvement}

Regional investigators and doctors from primary care facilities have had input into the study design, the development of the intervention tools, pilot testing phase and implementation of the intervention through roundtable and periodical national/regional review meetings. Participating doctors will be informed of study progress through monthly newsletters and progress reports. Patients with T2D in different areas have been interviewed for needs analysis at the preparatory and pilot phases, and will be interviewed again about implementation barriers and facilitators and the burden of their participation as part of a process evaluation at the end of the study. To encourage active engagement, participants receive their results from baseline and end-of-study assessments. The main results of the study will be disseminated to doctors and participants to boost community involvement in T2D management beyond the study.

\section{Ethics and dissemination}

Written approval from each participating site was granted by the local hospital research ethics committee and other relevant regional regulatory bodies. Signed informed consent is obtained from all trial participating doctors and patients prior to participant recruitment. Findings from this study will be widely disseminated through peerreviewed journals, conference presentations, social media and other mechanisms.

\section{DISCUSSION}

We outline the design for a large cluster clinical trial to evaluate the effectiveness of an innovative 4-in-1 T2D management model of care in China, to generate evidence for future scale up. Given the disparities in care under different contexts, the study areas are randomised according to stratification of developed or less-developed, urban or rural areas. The large sample size will enable us to appraise the effectiveness of the intervention overall and across each strata. Meanwhile, information collected on the barriers and facilitators of the intervention will better inform post-evaluation implementation and future scale up.

ROADMAP is complex in the intervention design per se and its implementation. We prepared for almost 2 years to develop and pilot the intervention as well as the project management and data collection system with the involvement of local doctors, patients and project managers. To make the 1-year intervention implemented successfully, we did not launch the project in 25 provinces (the plan was 24 provinces) in parallel, but launched one by one within a whole year, allowing lessons learnt to be transferred sequentially. Due to the limited research implementation experiences of most primary care investigators, it causes great challenges to ensure the quality of research implementation at the grassroots level. The use of two mobile phone-based project management and data collection systems (mEDC) helped to facilitate the implementation of the study with standard procedures and quality.

Intervention fidelity is another major challenge, especially considering the complexity of the ROADMAP, such as the number of study sites, and the shortage in qualified primary care workforce. mHealth technologymediated strategies have therefore been developed and applied to prevent and address inconsistencies occurring throughout the implementation. Besides, a governance system has been built up which includes provincial primary investigators and endorsed by the CDS. 
Reimbursement, or pay for performance, may affect the service providers' motivation and quality of care. It might be argued that if the reimbursement is only given to the intervention group, the effectiveness between the two arms would be incomparable. The study working group set a cap to the payment amount. On one hand, the reimbursement intends to serve as an additional workload compensation for the staff in the intervention groups. On the other hand, since it would be included as intervention-based cost, its cost-effectiveness may inform further policy adoption. During project implementation, local medical centres will determine the provision of reimbursements. The actual amount of this payment will be recorded in the project management system for health economics analysis.

To address the inconvenience and huge cost of centralised laboratory tests, we adopted a unified, portable, proven accurate instrument for measuring HbAlc, thus avoiding measurement error arising from the use of discrepant devices/systems. However, all other laboratory tests were performed by local county hospitals. The machines, methods and laboratory skills are different among hospitals although all of the routine sample (blood and urine) tests are under supervision and authorised by the government. We also collected the information of machines and methods for biochemical testing from all the participating hospitals.

China's health reform is still ongoing. Policy around the services delivery and financing of primary healthcare may encounter regional adjustment during the intervention period. We will record relevant policy changes but it still could potentially influence implementation and outcomes.

As all participants have been randomly recruited from the lists of those registered T2D in the communities, they are likely to be broadly representative of those with the condition in the real-world and thus enhance the generalisability of the results. We recognise, however, that our findings may not be extrapolated to those with undiagnosed or unregistered T2D.

We anticipate that ROADMAP would be implemented successfully, and the evaluation processes conducted with high quality. Most importantly, we hope this real-world trial could successfully contribute to the improvement of the management of T2D in China.

\footnotetext{
Author affiliations

${ }^{1}$ Department of Endocrinology and Metabolism, Shanghai Jiaotong University Affiliated Sixth People's Hospital, Shanghai, China

${ }^{2}$ Chinese Diabetes Society, Chinese Medical Association, Beijing, China

${ }^{3}$ Diabetes Group, The George Institute at Peking University Health Science Center, Beijing, China

${ }^{4}$ The George Institute for Global Health, University of New South Wales, Sydney, New South Wales, Australia

${ }^{5}$ Department of Endocrinology, Nanjing Medical University, Nanjing, China

${ }^{6}$ Department of Endocrinology, Sir Run Run Shaw Hospital, Zhejiang University

School of Medicine, Hangzhou, China

${ }^{7}$ Statistics and Data Group, The George Institute at Peking University Health Science Center, Beijing, China

${ }^{8}$ School of Computing, Beihang University, Beijing, China
}

Acknowledgements The authors acknowledge personnel who engaged in helping us accomplish all trial procedure. Thanks for the support from trial steering committee and efforts made by road to hierarchical diabetes management at primary care working group; all participating clinical research associates; investigators; doctors and patients, as well as those who helped to facilitate trial preparation; execution and evaluation. We thank Professor Craig Anderson for providing constructive guidance in writing this manuscript. We also thank Thomas Lung for proofreading the manuscript.

Contributors WJ and PZ conceived the project, designed the study, equally. WJ, PZ, ND, DZ, HL, YB and XL contributed to the design of the intervention and the evaluation. ND and PZ wrote the first draft of the manuscript. YL led the development of the electronic systems involved in road to hierarchical diabetes management at primary care intervention and trial management. XL was the statistician of the study. All authors contributed to the refinement of the study protocol and approved the final manuscript.

Funding This study receives its principle funding from following organisations: (1) Bethune Charity Foundation provides blood glucose (BG) monitoring and supporting decision-making system, BG monitoring equipment (smart BG meters) and consumables and project implementation fund required in the study (paththrough cost for county hospitals to support the intervention, data collection and performance incentives) as scheduled in accordance with the provisions of this agreement. (2) George Institute for Global Health provides financial and personnel support for, including but not limited to, training, institutional review board, project and data management, qualitative analysis, statistics and result publication.

Competing interests None declared.

Patient consent for publication Not required.

Provenance and peer review Not commissioned; externally peer reviewed.

Open access This is an open access article distributed in accordance with the Creative Commons Attribution Non Commercial (CC BY-NC 4.0) license, which permits others to distribute, remix, adapt, build upon this work non-commercially, and license their derivative works on different terms, provided the original work is properly cited, appropriate credit is given, any changes made indicated, and the use is non-commercial. See: http://creativecommons.org/licenses/by-nc/4.0/.

\section{REFERENCES}

1 International Diabetes Federation. IDF diabetes atlas. 8th edn Brussels, Belgium: International Diabetes Federation, 2017. http:// www.diabetesatlas.org

2 Global Burden of Metabolic Risk Factors for Chronic Diseases Collaboration. Cardiovascular disease, chronic kidney disease, and diabetes mortality burden of cardiometabolic risk factors from 1980 to 2010: a comparative risk assessment. Lancet Diabetes Endocrinol 2014;2:634-47.

3 Seuring T, Archangelidi O, Suhrcke M. The economic costs of type 2 diabetes: a global systematic review. Pharmacoeconomics 2015;33:811-31.

$4 \mathrm{Li} \mathrm{X,} \mathrm{Lu} \mathrm{J,} \mathrm{Hu} \mathrm{S,} \mathrm{et} \mathrm{al.} \mathrm{The} \mathrm{primary} \mathrm{health-care} \mathrm{system} \mathrm{in} \mathrm{China.} \mathrm{The}$ Lancet 2017;390:2584-94.

5 Huo L, Shaw JE. Type 2 diabetes: challenges facing GPs. Fam Med Community Health 2018;6:26-31.

6 Jin Y, Zhu W, Yuan B, et al. Impact of health workforce availability on health care seeking behavior of patients with diabetes mellitus in China. Int J Equity Health 2017;16.

7 Ministry of finance, PRC (2019) report on the execution of the central and local budgets for 2018 and on the draft central and local budgets for, 2019. Available: http://www.xinhuanet.com/english/ 2019-03/17/c_137901687.htm [Accessed 25 Jun 2019].

8 Ministry of Health, PRC. Opinions on promoting the essential public health services gradually equalization, 2009. Available: http://www. nhc.gov.cn/wjw/gfxwj/201304/6cf4917e8af44fe28ac655f215f8e4ed. shtml [Accessed 25 Jun 2019].

9 People's Republic of China health system review. Manila : WHO Regional Office for the Western Pacific; 2015. (pg.20-21:139-42.

10 National Health and Family Planning Commission of the People's Republic of China. China health and family planning statistical yearbook 2017. Beijing: Peking Union Medical College Publishing House, 2018.

112016 health system reform progress monitoring report, 2017. Available: http://www.nhc.gov.cn/tigs/ygjb/201707/72dbee11426a 48cfbe66325c20099c5f.shtml?from=singlemessage\&isappinstalled= 0\#10006-weixin-1-52626-6b3bffd01fdde4900130bc5a2751b6d1. [Accessed 20 Sep 2019]. 
$12 \mathrm{Xu}$ Yet al. Prevalence and control of diabetes in Chinese adults. JAMA 2013;310:948-59.

13 Ji L, Hu D, Pan C, et al. Primacy of the 3B approach to control risk factors for cardiovascular disease in type 2 diabetes patients. Am J Med 2013;126:925.e11-925.e22.

14 Davidson JA. The increasing role of primary care physicians in caring for patients with type 2 diabetes mellitus. Mayo Clinic Proceedings 2010;85:S3-4

15 International diabetes Federation. recommendations for managing type 2 diabetes in primary care, 2017. Available: www.idf.org/ managing-type2-diabetes [Accessed 25 Jun 2019].

16 Meng Q, Mills A, Wang L, et al. What can we learn from China's health system reform? BMJ 2019;।.

17 Yuan B, Balabanova D, Gao J, et al. Strengthening public health services to achieve universal health coverage in China. BMJ 2019;33.

18 Yue J, Mao X, Xu K, et al. Prevalence, awareness, treatment and control of diabetes mellitus in a Chinese population. PLoS One 2016;11:e0153791.

19 Ahmad NS, Islahudin F, Paraidathathu T. Factors associated with good glycemic control among patients with type 2 diabetes mellitus. J Diabetes Investig 2014;5:563-9.

20 Lou Q, Wu L, Dai X, et al. Diabetes education in mainland China-A systematic review of the literature. Patient Educ Couns 2011;85:336-47.

21 Choi TST, Davidson ZE, Walker KZ, et al. Diabetes education for Chinese adults with type 2 diabetes: a systematic review and metaanalysis of the effect on glycemic control. Diabetes Res Clin Pract 2016;116:218-29.

22 Liu Y, Zhong L, Yuan S, et al. Why patients prefer high-level healthcare facilities: a qualitative study using focus groups in rural and urban China. BMJ Global Health 2018;3:e000854.

23 State Council, PRC. Guiding opinions of the general office of the state Council on Propelling building of grading diagnosis and treatment system, 2015. Available: http://www.gov.cn/zhengce/ content/2015-09/11/content_10158.htm [Accessed 25 Jun 2019]

24 CPC Central Committee, State Council. The plan for "Healthy China 2030", 2016. Available: http://www.gov.cn/xinwen/2016-10/25/ content_5124174.htm [Accessed 25 Jun 2019].

25 Johnston L, Zemanek J, Reeve MJ, et al. The evidence for using mHealth technologies for diabetes management in low- and middleincome countries. J Hosp Manag Health Policy 2018;2.
26 Reynolds R, Dennis S, Hasan I, et al. A systematic review of chronic disease management interventions in primary care. BMC Fam Pract 2018;19.

27 Mitchell GK, Burridge L, Zhang J, et al. Systematic review of integrated models of health care delivered at the primary-secondary interface: how effective is it and what determines effectiveness? Aust $J$ Prim Health 2015;21:391.

28 Morrison F, Shubina M, Turchin A. Encounter frequency and serum glucose level, blood pressure, and cholesterol level control in patients with diabetes mellitus. Arch Intern Med 2011;171:1542.

29 Coupe N, Peters S, Rhodes S, et al. The effect of commitmentmaking on weight loss and behaviour change in adults with obesity/overweight; a systematic review. BMC Public Health 2019;19.

30 Ralston JD, Rutter CM, Carrell D, et al. Patient use of secure electronic messaging within a shared medical record: a crosssectional study. J Gen Intern Med 2009;24:349-55.

31 Bredfeldt CE, Compton-Phillips AL, Snyder MH. Effects of between visit physician-patient communication on diabetes recognition program scores. Int J Qual Health Care 2011;23:664-73.

32 Song S, Yuan B, Zhang L, et al. Increased inequalities in health resource and access to health care in rural China. Int $J$ Environ Res Public Health 2018;16:49.

33 Zhang T, Xu Y, Ren J, et al. Inequality in the distribution of health resources and health services in China: hospitals versus primary care institutions. Int J Equity Health 2017;16

34 Chinese Diabetes Society. National Office for Primary Diabetes Care. National guidelines for the prevention and control of diabetes in primary care(2018). Zhonghua Nei Ke Za Zhi 2018;57:885-93.

35 National Bureau of statistics of China. China statistical Yearbook 2017, 2018. Available: http://www.stats.gov.cn/tjsj/ndsj/2017/ indexeh.htm [Accessed 25 Jun 2019].

36 Zhou R, Wang W, Song Z-X, et al. Evaluation of a new hemoglobin A1c analyzer for point-of-care testing. J Clin Lab Anal 2018;32:e22172.

37 Ji L, Zhang P, Weng J, et al. Observational registry of basal insulin treatment (orbit) in patients with type 2 diabetes uncontrolled by oral hypoglycemic agents in China-Study design and baseline characteristics. Diabetes Technol Ther 2015;17:735-44. 